\title{
BMJ Open Increasing the uptake of long-acting reversible contraception in general practice: the Australian Contraceptive ChOice pRoject (ACCORd) cluster randomised controlled trial protocol
}

\author{
Danielle Mazza, ${ }^{1}$ Kirsten Black, ${ }^{2}$ Angela Taft, ${ }^{3}$ Jayne Lucke, ${ }^{4}$ Kevin McGeechan, ${ }^{5}$ \\ Marion Haas, ${ }^{6}$ Heather McKay, ${ }^{7}$ Jeffery $F$ Peipert ${ }^{8}$
}

To cite: Mazza D, Black K, Taft $A$, et al. Increasing the uptake of long-acting reversible contraception in general practice: the Australian Contraceptive ChOice pRoject (ACCORd) cluster randomised controlled trial protocol. BMJ Open 2016;6:e012491.

doi:10.1136/bmjopen-2016012491

- Prepublication history for this paper is available online. To view these files please visit the journal online (http://dx.doi.org/10.1136/ bmjopen-2016-012491).

Received 4 May 2016 Revised 27 July 2016 Accepted 9 September 2016

CrossMark

For numbered affiliations see end of article.

Correspondence to Professor Danielle Mazza; Danielle.Mazza@monash.edu

\section{ABSTRACT}

Introduction: The increased use of long-acting reversible contraceptives (LARCs), such as intrauterine devices and hormonal implants, has the potential to reduce unintended pregnancy and abortion rates. However, use of LARCs in Australia is very low, despite clinical practice guidance and statements by national and international peak bodies advocating their increased use. This protocol paper describes the Australian Contraceptive ChOice pRojet (ACCORd), a cluster randomised control trial that aims to test whether an educational intervention targeting general practitioners (GPs) and establishing a rapid referral service are a cost-effective means of increasing LARC uptake.

Methods and analysis: The ACCORd intervention is adapted from the successful US Contraceptive CHOICE study and involves training GPs to provide 'LARC First' structured contraceptive counselling to women seeking contraception, and implementing rapid referral pathways for LARC insertion. Letters of invitation will be sent to 600 GPs in South-Eastern Melbourne. Using randomisation stratified by whether the GP inserts LARCs or not, a total of 54 groups will be allocated to the intervention (online 'LARC First' training and rapid referral pathways) or control arm (usual care). We aim to recruit 729 women from each arm. The primary outcome will be the number of LARCs inserted; secondary outcomes include the women's choice of contraceptive method and quality of life (Short Form Health Survey, SF-36). The costs and outcomes of the intervention and control will be compared in a cost-effectiveness analysis.

Ethics and dissemination: The ACCORd study has been approved by the Monash University Human Research Ethics Committee: CF14/3990-2014002066 and CF16/188-2016000080. Any protocol modifications will be communicated to Ethics Committee and Trial Registration registry. The authors plan to disseminate trial outcomes through formal academic pathways comprising journal articles, nation and international conferences and reports, as well as

\section{Strengths and limitations of this study}

- The Australian Contraceptive ChOice pRoject (ACCORd), building on an already successful US study-The Contraceptive CHOICE Project, is trialling a multifaceted practice-based intervention. It is the first Australian trial to test this type of intervention and measure the cost-effectiveness of long-acting reversible contraceptives (LARCs) in this country.

- The trial may provide a model for both education of general practitioners (GPs) and a rapid referral system for LARCs that can be delivered in other Australian settings.

- The trial may provide insight into GP characteristics that are associated with change in practice following the educational intervention.

- We recognise that the participating GPs may not be representative of the Australian GP population: the trial is being conducted in a narrow setting focused on southeast Melbourne; and participating GPs who are interested in LARCs may be over-represented.

- The study does not address GP experience and skill in delivering LARC methods, as GPs are not being trained to insert LARCs, so this remains a potential barrier for GPs.

using more 'popular' strategies including seminars, workshops and media engagements.

Trial registration number: ACTRN12615001346561.

\section{INTRODUCTION}

Approximately $41 \%$ of pregnancies occurring worldwide are unintended. ${ }^{1}$ Reported rates in the USA and the UK are $49 \%^{2}$ and $41 \%{ }^{3}$ respectively. In Australia, there is no reliable data concerning women's pregnancy 
intentions; however, a cross-sectional survey of a nationally representative sample of Australian women found that around $51 \%$ had experienced an unintended pregnancy in their lifetime. ${ }^{4}$

Unintended pregnancies have significant consequences both socially and economically. In the UK, it is estimated that the healthcare and social costs of unintended pregnancies can be as much as $£ 1$ billion per year. ${ }^{5}$ Estimates for the USA put the cost of abortions, miscarriage management, antenatal care, births and infant medical care for unintended pregnancies at around US $\$ 9.6-\mathrm{US} \$ 12.6$ billion per annum. ${ }^{6}$

While a number of safe contraceptive methods are currently available to women, the effectiveness of different methods in preventing pregnancy varies widely. Apart from permanent sterilisation methods (such as vasectomy and tubal ligation), it is generally accepted that long-acting reversible contraceptives (LARCs), including intrauterine devices (IUDs) and progestogen implants, are the most effective at preventing pregnan$\operatorname{cies}^{7}$ due, in part, to their minimal adherence requirements and long duration. In contrast, other methods, such as the oral contraceptives, vaginal ring and condoms (ie, short-acting reversible contraceptives or SARCs), can be very effective at preventing pregnancies, but only when strict adherence is consistently maintained. Given this, it is not surprising that unintended pregnancies still occur during contraception use-in the USA, around $48 \%$ of unintended pregnancies are associated with contraception use during the month of conception. ${ }^{8}$ The incidence of unintended pregnancy varies, however, according to the contraceptive method used with around $0.05-0.8 \%$ of women conceiving during first year use of a LARC method compared with around $3-32 \%$ of women using other reversible methods. ${ }^{7}$ Findings from the US Contraceptive CHOICE Project indicate that the risk of contraceptive failure among women who use oral contraceptives, a transdermal patch or a vaginal ring is 20 times greater than the risk among women who use LARCs. ${ }^{9}$

Cost-effectiveness analyses of different contraceptive methods have also favoured LARC use. A UK study reported that despite the high start-up costs related to health service delivery, all LARC methods were more effective and less costly than the combined oral contraceptives. ${ }^{10}$ Similar findings are reported in the USA where, apart from the irreversible methods, LARC methods have been found to be the least expensive and most effective contraceptive method. ${ }^{11}$ From a costsavings perspective, if $10 \%$ of US women aged between 20 and 29 years switched to a LARC method, then approximately US $\$ 375$ million per year could potentially be saved from the cost of unintended pregnancies and contraception use. ${ }^{12}$

Despite their effectiveness in preventing pregnancies, and evidence of their cost-effectiveness in numerous countries, a number of barriers to LARC uptake have been identified. In studies examining patients' understanding of LARCs, specific details and knowledge of the benefits of LARCs were found to be low among women. ${ }^{13}{ }^{14}$ Additionally, many women still hold myths and misconceptions about the side effects and risks associated with LARCs. ${ }^{15}$ In particular, negative views about IUDs causing infection and infertility have persisted despite evidence that modern forms are safe for use in all women (including young nulliparous women), and are highly effective at preventing unintended pregnancies without impacting future fertility. ${ }^{16} 17$

Health practitioners' knowledge and experience of LARC methods is also lacking. A UK study exploring the views of general practitioners (GPs) and nurse practitioners reported that when asked about an ideal contraceptive for a 19-year-old woman with no children, only $27 \%$ would consider an implant and $2 \%$ an IUD, compared with $85 \%$ and $80 \%$ who would consider combined oral contraceptives and condoms, respectively. ${ }^{18}$ These practitioners identified lack of skill in delivering the methods $(61 \%)$, concern over irregular vaginal bleeding $(50 \%)$ and concern about high method discontinuation rates $(21 \%)$ as their key issues around LARC provision.

GPs' views on contraception can also influence the advice they give to potential users, what contraceptive methods are made available to women, and the type of contraception selected by patients. ${ }^{18-21}$ Although a recent review of barriers preventing the widespread use of IUDs among nulliparous women identified healthcare provider, health system and user barriers, the beliefs of healthcare providers were found to have the most profound effect on the uptake of IUDs. ${ }^{22}$ An Australian survey of women presenting for IUD insertion found that $18 \%$ had been told by either a health professional, friend or family member that it was not a suitable method for them, despite these women meeting the appropriate medical eligibility criteria. ${ }^{23}$

Several interventions to increase LARC uptake have been tested. Most of these have focused on one form of LARC only (mainly IUDs) or have targeted either providers or women, but not both. A Cochrane review of interventions reported that counselling was effective in increasing the uptake of IUDs. ${ }^{24}$ Women who were (1) provided with contraceptive counselling and referrals by trained community-based workers; (2) provided with antenatal contraceptive counselling; or (3) provided with postnatal couple contraceptive counselling prior to discharge, were found to have a higher uptake of copper IUDs compared with those who did not receive any form of counselling. Additionally, acceptability of levonorgestrel implants is enhanced by effective counselling, particularly preinsertion, even in the presence of side effects. ${ }^{25} 26$

Counselling and providing LARCs at no cost has also proven to be an effective intervention in several settings. In a study involving women who had abortions, a significant increase (6.3-36.3\%) in the uptake of levonorgestrelcontaining intrauterine systems (LNG-IUS) was reported among women who received the interventions 
postabortion compared with those in the control group. ${ }^{27}$ Furthermore, $89 \%$ of these women were still using LNG-IUS after 6 weeks, with only a slight decrease after 6 months $(81 \%)$. A follow-up study on the same cohort of women reported that those who had used LARCs had significantly lower return rates for repeat abortion compared with those who had used other contraceptive methods. ${ }^{28}$

In response to the increasing number of unintended pregnancies in the USA, and the low uptake of LARCs, the Contraceptive CHOICE Project (CHOICE) started in 2007. The primary aim of this prospective cohort study was to reduce the number of unintended pregnancies by providing contraception at no cost to women in St Louis, Missouri, and promoting the use of LARCs through structured contraceptive counselling. ${ }^{29}$ During the 5-year study period, a total of 9526 women aged between 14 and 45 years were enrolled. Reported outcomes include a higher rate of LARC uptake compared with the reported national rate; $;^{30}$ a significant reduction in abortion rates compared with the regional and national rates; ${ }^{30}$ lower birth rates among women aged 15-19 years compared with the national rate; $;^{30}$ and high rates of continuation for IUDs and implant at 24 months. ${ }^{31}$ These outcomes not only support the use of LARCs for reducing unintended pregnancies, but reinforce the importance of educating women about LARCs as a way of increasing the rate of LARC uptake.

The efficacy of LARC effectiveness counselling even when usual contraceptive costs are maintained has also been tested in the USA. Harper et a $\vec{l}^{2}$ conducted a cluster randomised control trial aimed at improving the knowledge and counselling, and insertion skills of providers at 40 reproductive health clinics. Providers in half of the clinics received a training intervention designed to improve their knowledge and counselling, and insertion skills, while the other half were instructed to provide usual care. Reported outcomes include: of the 1500 women who participated in the study, more women in the intervention arm (28\%) selected LARCs during their contraceptive visit than those in the control arm $(17 \%)$ where usual care was provided. ${ }^{32}$

No interventions designed to increase LARC uptake among Australian women have been tested. The prescription and use of LARCs in Australia is very low despite clinical practice guidance and statements by peak bodies advocating an increase in the use of LARCs. ${ }^{33}$ In a survey of women aged $16-59$ years conducted in 2001-2002, <3\% reported using LARCs (IUDs $1.2 \%$; implants $1.1 \%) .{ }^{34}$ Similarly, data from the household, income and labour dynamics in Australia survey in 2005 indicated that $<5 \%$ of women who were at risk of pregnancy were using LARCs $(1.6 \%$ were using an IUD and $2.8 \%$ were using an implant). ${ }^{35}$ Analysis of Australian general practice consultations using data from the Bettering the Evaluation and Care of Health $(\mathrm{BEACH})$ programme indicated that only $6.9 \%$ of all contraception consultations involved LARCs. ${ }^{19}$ In this country, general practice is the 'first line' in the delivery of contraception to women and the management of sexual and reproductive health concerns; therefore, interventions aimed at increasing LARC uptake in the general practice setting are critical and likely to have the most impact.

ACCORd is an Australian adaption of the US Contraceptive CHOICE Project. The study aims to test whether an educational intervention targeting GPs and establishing a fast-track referral process are a costeffective means of increasing the uptake of LARCs among Australian women. This intervention has the potential to increase the uptake of LARCs among Australian women by addressing patient, practitioner and system barriers, and reduce the rates of unintended pregnancies in Australia.

\section{METHODS AND ANALYSIS}

\section{Study design}

This study is a CONSORT compliant ${ }^{36}$ cluster randomised controlled trial (RCT) of a complex intervention that involves training GPs to provide 'LARC First' structured contraceptive counselling and implementing rapid referral pathways to LARC insertion. Individual GPs will be the unit of randomisation; however, interventions and analyses will target the cluster level (GP) and individual level (women).

The trial will comprise two arms: (1) GPs who have been trained to deliver 'LARC First' structured contraceptive counselling and have rapid referral pathways for LARC insertion, and (2) GPs who will deliver usual contraceptive care. Women (patients of the GPs) will be recruited to each arm. Figure 1 depicts the trial flow chart.

\section{Randomisation}

A statistician not directly involved with the recruiting process, and blinded to the identity of the GPs, will allocate eligible GPs to the intervention or control arm using a randomisation sequence generated using permuted blocks, with block sizes of 4, 6 and 8 , stratified by whether the GP inserts LARCs (IUDs/implants) or not. The computer program Rand.exe ${ }^{37}$ will be used to generate the randomisation sequence.

\section{Setting}

The trial is being conducted among GPs who practice in Melbourne, Australia. Recruitment is scheduled to begin in May 2016 and finish in April 2017.

\section{Inclusion and exclusion criteria}

\section{General practitioners}

GPs are eligible to participate if they work three or more sessions per week, are based at a computerised practice, and have a receptionist who can assist with the recruitment of women (patients of the GP). To avoid contamination due to cross-over effects, only one GP per practice will be included. 
Figure 1 Trial flow chart. GP, general practitioner; LARC, long-acting reversible contraceptive; SARC, short-acting reversible contraceptive.

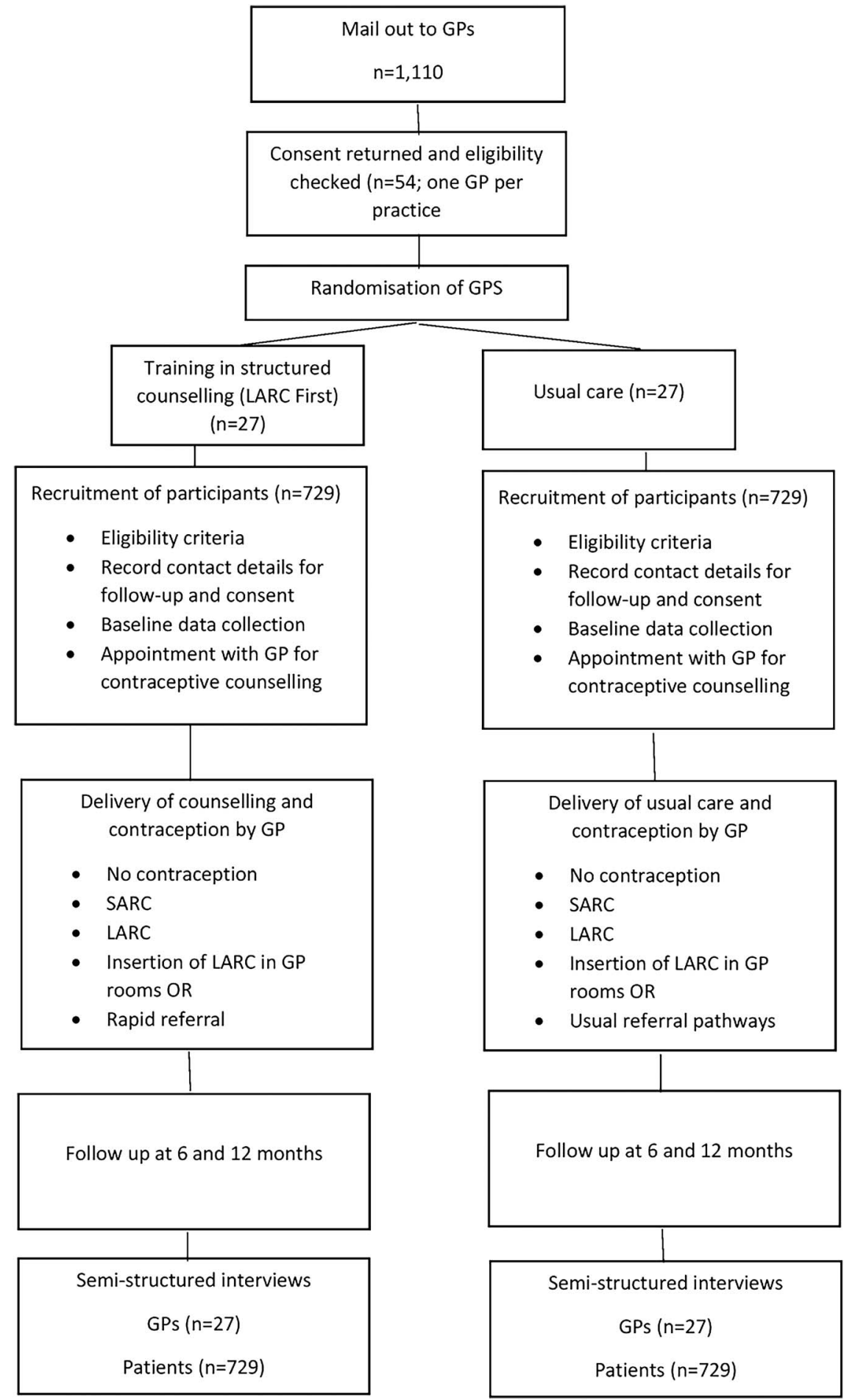

\section{Women}

Women are eligible to participate if they are aged between 16 and 45 years, have been sexually active with a male partner in the past 6 months or anticipate sexual activity in the next 6 months, have not undergone a tubal ligation or hysterectomy, have partner(s) who have not undergone a vasectomy, are not pregnant or have no desire to become pregnant in the next year, speak proficient English, and are interested in discussing contraception or in starting a new, reversible contraceptive method.

\section{Participant and recruitment procedures}

\section{General practitioners}

GPs in southeast Melbourne will be mailed an invitation letter, explanatory statement and expression of interest form. Non-responders will be sent a follow-up letter after 2 weeks, and receive a reminder telephone call after another 2 weeks until recruiting has achieved target numbers. If necessary, the study will also be advertised in relevant newsletters. GPs who return the expression of interest form will receive a practice visit by an 
ACCORd researcher when a signed consent form will be obtained. Participating GPs will be assigned 40 Category 1 Royal Australian College of General Practitioners (RACGP) Quality Assurance and Continuing Professional Development points for study participation, and receive $\$ A 500$ as reimbursement for time spent on the study.

\section{Women}

Women (16-45 years) attending for a consultation with their GPs (participating in the trial) will be invited by receptionists to complete an eligibility survey (including contact details for eligible women) on an iPad in the waiting room. The research team will then contact the eligible women by telephone to obtain consent, undertake baseline data collection. Women will be asked to make an appointment to see their GP and a researcher will follow-up within 1 week to ensure an appointment has been made. Consent will also be obtained from participants to access their Medicare (Medicare Benefits Scheme (MBS) and Pharmaceutical Benefits Scheme (PBS)) records for the purposes of a health economic evaluation. Medicare data are highly accurate because government payments by Medicare through the MBS and PBS ensure that providers and patients are incentivised to keep accurate records.

\section{Intervention}

GPs in the intervention arm will be trained to deliver structured contraceptive counselling with an emphasis on the effectiveness of LARCs. Materials from the 'LARC First' online training site of the Contraceptive CHOICE Project ${ }^{38}$ were adapted to the Australian context with input from an advisory group comprising the project investigators, GPs and consumers. Training will be delivered online with additional practice visits, email and telephone support available. To ensure fidelity of the counselling, within 4 weeks of recruitment to the trial, random visits to all GPs will be conducted. During this visit, a researcher (blinded to the allocation of the GP to intervention or control arm) will observe a single consultation and complete a checklist. It is anticipated that a higher proportion of GPs in the intervention arm will deliver LARC-First structured counselling with the intent that was envisioned by the ACCORd team. This fidelity check will provide us with insights that will help explain the trial outcomes, that is, a change in LARC uptake.

Structured contraceptive counselling ${ }^{39}$ will be delivered to the participating women by the intervention trained GPs. These GPs will provide a non-biased, scripted description of all available contraceptive methods, with particular reference to the safety and efficacy of each method. They will also collect clinical information from the women to identify any contraindications or conditions that may influence the choice of contraception-women will be able to choose their contraception method provided that it is not medically contraindicated. The GP will then screen the woman for pregnancy (urine pregnancy test) and chlamydia (according to clinical practice guidelines published by the RACGP).$^{40}$ On ruling out pregnancy, the GP will either: (1) provide a prescription for the method of choice; (2) offer 'same day' insertion of the LARC method, or at a subsequent time at the GP clinic; or (3) provide an appointment for insertion of the LARC method at one of the insertion clinics. Emergency contraception will be offered to women who have had recent unprotected intercourse, while 'quick start' contraception (ie, starting contraception at any time rather than at the start of the next menstrual cycle) will be offered to women (as per the Faculty of Sexual and Reproductive Healthcare guidelines ${ }^{41}$ ) in cases where pregnancy cannot yet be ruled out. These women will also be offered the opportunity to return in 34 weeks for a LARC insertion (and a repeat pregnancy test).

A rapid referral pathway to a LARC insertion clinic will be implemented for instances where the intervention group GP does not undertake insertions in their own rooms-GPs will book appointments for women to have a LARC insertion at study-specific LARC insertion clinics provided in the rooms of local private gynaecologists. A reimbursement of $\$ A 300$ per $3 \frac{1}{2}$ hours will be provided to doctors providing these LARC insertion clinics. Doctors in the control group will perform their usual contraceptive care and will not have access to the rapid referral clinics.

\section{Control group}

GPs in the control group will provide usual contraceptive care to women recruited to this arm. At the conclusion of the trial, the control group of GPs will be invited to undertake the online 'LARC First' training.

\section{Data collection and measures}

Description of groups

Characteristics of participating GPs (eg, age, years of general practice experience, and education in women's health and contraception) will be recorded via a survey administered at baseline. Data will be used to check the extent to which randomisation creates equivalence across the two groups-intervention and control arms.

\section{Outcome evaluation}

We will use the Contraceptive CHOICE Project data collection surveys with participating women and modify these for the Australian context. ${ }^{28}$ We will also use the Short Form Health Survey (SF-36) ${ }^{42}$ to measure quality of life, and the Health Literacy Questionnaire (HLQ) ${ }^{43}$ which is a validated tool designed to identify skills and knowledge which determine the motivation and ability of individuals to access healthcare and maintain good health. Figure 2 describes the variables recorded throughout the trial. 
Data collection from participating women will occur as follows:

- At baseline: by clinical trial coordinator via telephone interview;

- At the contraceptive counselling consultation: by GP using a standardised data collection form;

- At the time of contraception uptake. by GP or health professional using a standardised data collection form;

- At 6 and 12 months postenrolment: by clinical trial coordinator via telephone interview.

The primary outcome is the number of LARCs inserted; secondary outcomes include the women's choice of contraceptive method and quality of life (SF-36). Other data collected, such as time from consultation to insertion of LARC (see figure 2) will be used to investigate mediating variables.

All hard copy forms will be kept in locked cabinet in the project manager's office. Data collected from GPs and women participants will be de-identified and kept as electronic files in password-protected project file on the University ' $S$ ' drive. Only ACCORd investigators and project team will have access to these files.

\section{Process evaluation}

The realistic evaluation model ${ }^{44}$ will be used to understand 'what works for whom and in what circumstances'. This evaluative framework examines context, mechanism and outcomes and will consist of (in addition to the 6-month and 12-month data):

- GPs completing a Knowledge, Attitudes, and Practices (KAP) survey at baseline and at the 12-month interview;

- GPs (intervention arm) completing short encounter forms describing the outcomes of the structured contraceptive counselling;

- Fidelity checks to assess GP participants in the intervention arm of the ACCORd project are delivering LARC-First structured counselling with the intent that was envisioned by the ACCORd team;

- Semistructured telephone interviews with up to 20 women (sampled for maximum diversity in age, parity and socioeconomic status) from each arm after the 12-month assessment to investigate their experiences of receiving the intervention or usual care and perceived outcomes;

- Semistructured telephone interviews with all GPs (both arms) to assess their perceptions of the research and intervention process and the impact on their practice, both positive and negative.

\section{Economic evaluation}

An economic evaluation will be undertaken comparing the intervention and control arms in a cost-effectiveness analysis (CEA). A trial-based analysis will be followed by the construction of a decision model to extrapolate future costs and benefits beyond the completion of the trial. The overall perspective used will be that of the health system. Costs will include the design and implementation of the GP education tool, direct and indirect costs of health service use, and the purchase and administration of contraceptive products. Outcomes will be measured in terms of number of LARCs inserted, type of contraception chosen, health-related quality of life (HRQoL) and quality-adjusted life years (QALYs) gained. HRQoL will be measured using the SF-36 and QALYs gained will be estimated using the SF-6D, which allows the calculation of QALYs from the SF-36 for use in CEA.

\section{Sample size and power calculation}

The study requires 24 GPs and 24 women per GP in each of the two study arms (intervention and control) to detect a change in the LARC insertion rate from $10 \%$ to $20 \%$, with $80 \%$ power and a significance level of $5 \%$. This will allow for the stratification by whether or not GPs insert LARCs (IUDs or implants) and a clustering effect (intracluster correlation (ICC)) of 0.05 . This corresponds to the maximum ICG for variables associated with GP-patient encounters in a recent cluster RCT ${ }^{45}$ and other GP-specific studies. ${ }^{46}$

ACCORd will recruit a total of 27 GPs and 27 women per GP in each of the two study arms to allow for up to a $10 \%$ drop-out among $\mathrm{GPs}^{45}$ and a $10 \%$ drop-out among women. This low drop-out rate among the women has been assumed because the primary outcome (LARC insertion) will be scheduled soon after the consultation and the data for this outcome will be derived from the GP's standardised data collection form, not participant follow-up.

\section{Analyses}

\section{Description of groups}

We will summarise the demographic characteristics of GPs and women for both groups with counts and proportions, or means and SDs, where appropriate.

\section{Outcome evaluation}

The $\chi^{2}$ test, adjusted for clustering ${ }^{47}$ and stratification by whether or not the GP inserts LARCs, will be used for the primary analysis to compare the proportion of women who have had a LARC inserted. This approach will also be used to analyse the binary secondary outcomes. Exploratory analyses of possible mediating variables will be carried out using binary regression models with generalised estimating equations and robust SEs to account for clustering. We will use multiple imputation and sensitivity analyses to explore the impact of missing data.

\section{Process evaluation}

We will compare knowledge scores at baseline and at 12 months among GPs in the intervention group using the paired t-test, and individual items will be compared using McNemar's test. The two sample t-test, and $\chi^{2}$ test, will be used to compare responses at 12 months between GPs in the intervention and control groups. 


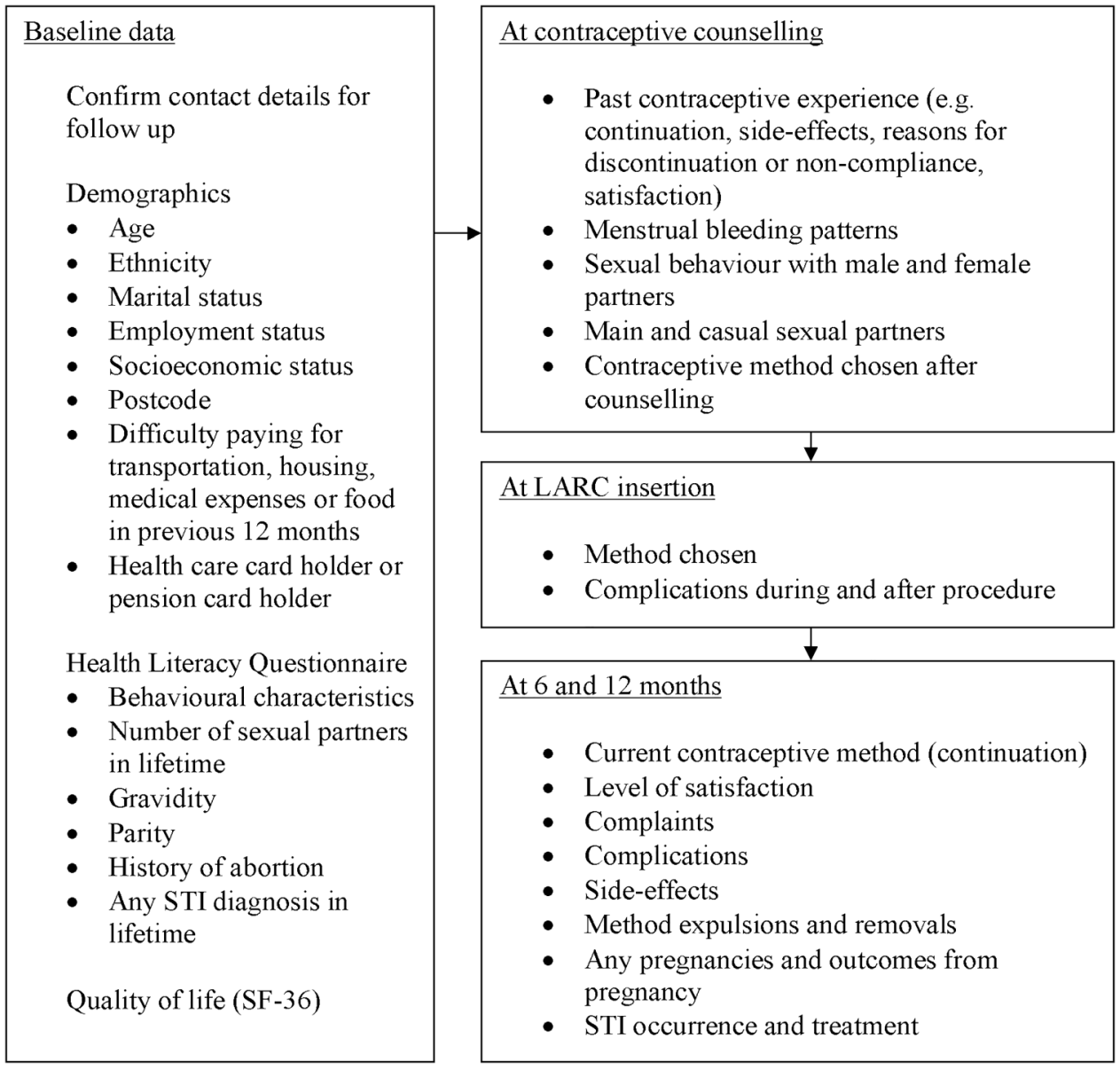

Figure 2 Variables recorded throughout the study. LARC, long-acting reversible contraceptive; STI, sexually transmitted infection.

Qualitative data will be thematically analysed using the computer software package NVivo.

\section{Economic evaluation}

Costs: The direct costs of the intervention and control arms will be measured as the costs of consultations with GPs and other medical practitioners and the costs of the contraceptive products, including their administration, where appropriate. Analysis of participants' Medicare (MBS and PBS) records will allow for an accurate estimate of medical service and pharmaceutical usage and the associated costs. Indirect costs will be measured in terms of travel time and time away from work for women.

Outcomes: The primary outcome for the trial-based CEA will be the number of QALYs gained. QALYs will be estimated using the SF-6D, a classification for describing health derived from a selection of SF-36 items. It is composed of six multilevel dimensions.

The results of the primary CEA will be reported as the cost/QALY gained. Cost-effectiveness will also be reported in terms of cost/change in HRQoL (measured using the SF-36 $6^{42}$ ), number of LARC inserted and type of contraception chosen. All cost-effectiveness results will be presented as net costs and benefits for the intervention arm versus the control arm at 12 months.
The costs of each arm will take into account any costsavings due to avoided unwanted pregnancies. Mean estimates of costs will be used and CIs generated by bootstrapping the data. The robustness and validity of the CEA will be explored using sensitivity analysis.

A modelled analysis is required to take into account costs and outcomes beyond the period of the trial. This will be informed by the outcomes and resource use observed during the trial, and extrapolated using data from the literature and other published sources. In addition to estimating outcomes in terms of QALYs, this analysis will also be able to assess the impact of the intervention on longer term consequences such as unwanted pregnancies avoided and abortions avoided. This analysis will enable account to be taken of the long-term impact on the resource use and costs associated with LARC (which are typically more expensive 'up-front' but much less expensive over time than SARC), as well as longer term impacts such as side effects, discontinuations in contraceptive use (including LARC) and pregnancy rates.

\section{Participant data and study management}

All participants (GPs and women) will be allocated a unique code. Survey data will be entered into a REDCap database; semistructured interview data will be audio 
recorded, transcribed and stored as MS Word documents. A Data Monitoring Committee will monitor trial recruitment, outcomes and adverse events. Comprising of three members (a statistician and two academic GP/ lecturers) external to the ACCORd project, this committee will meet every 3 months from initiation of recruiting until completion of the trial.

\section{Trial status}

This trial is registered with the Australian New Zealand Trials Registry ACTRN12615001346561. Recruitment will start May 2016: no data collection has started.

\section{DISCUSSION}

There is a pressing need to reduce unintended pregnancies and the associated social and economic consequences, and evidence indicates that increasing the uptake of LARC can play a substantial role in addressing this public health issue. ${ }^{21}$ The Contraceptive CHOICE Project, conducted in the USA, provided comprehensive data on the effectiveness of LARC focused information for women and the relative efficacy of LARC in pregnancy prevention-the study also eliminated the barrier of method cost. ${ }^{29}{ }^{30}$ In Australia, national surveys have indicated a low uptake of LARC methods among reproductive aged women. ${ }^{34} 35$ Strategies to increase Australian women's access to LARCs are important in addressing this pattern, and interventions that provide women with greater contraceptive choice will give them greater autonomy over their reproductive lives.

ACCORd aims to evaluate a complex intervention that involves training GPs to provide 'LARC First' structured contraceptive counselling and implementing rapid referral pathways to LARC insertion. This intervention addresses a number of potential barriers to LARC uptake in Australia. Through education, practitioners will receive knowledge and training for the delivery of accurate evidence-based information to women during contraceptive counselling. Women will therefore be better informed when choosing contraception, and more able to make choices that suit their wants and needs. Further, the provision of rapid referral pathways for LARC insertion provides better access to the timely LARC insertion. These approaches address practitioner, patient and systems barriers to LARC insertion and therefore this intervention has the potential to increase the uptake of LARCs among Australian women and reduce the rates of unintended pregnancies in Australia.

Currently there is limited Australian information about the economic cost of unintended pregnancy, or the costeffectiveness of LARCs. The ACCORd economic evaluation (one component of the study) accounts for the cost structure of the Australian health system, including issues such as reimbursement by the MBS and PBS and out-of-pocket costs to women. It will demonstrate where the burden of costs falls in increasing the use of LARCs (individuals vs the health system), as well as any potential cost-savings from using LARCs both for government and women. Further, the study will show the benefits to women of using LARCs not just in terms of reducing unplanned pregnancies, but also in terms of their quality of life.

Based on the trial results, which will include both the effectiveness of the intervention at increasing the rate of LARC uptake and a CEA of the intervention, this study will help evaluate the advantages of rolling out the intervention more widely. It also has the potential to make important recommendations about how to improve the current Australian system concerning the provision of LARCs. Finally, the intervention will contribute to national and international work concerning interventions and strategies to increasing the uptake of LARCs, and consequently reduce unintended pregnancy rates.

\section{Author affiliations}

${ }^{1}$ Department of General Practice, Monash University, Notting Hill, Victoria, Australia

${ }^{2}$ Department of Obstetrics, Gynaecology \& Neonatology, University of Sydney, Central Clinical School, Royal Prince Alfred Hospital, Sydney, New South Wales, Australia

${ }^{3}$ Judith Lumley Centre, La Trobe University, Melbourne, Victoria, Australia ${ }^{4}$ Australian Research Centre for Sex, Health and Society (ARCSHS), La Trobe University, Melbourne, Victoria, Australia

${ }^{5}$ School of Public Health, University of Sydney, Sydney, New South Wales Australia

${ }^{6}$ Centre for Health Economics Research and Evaluation (CHERE), University of Technology Sydney (UTS), Sydney, New South Wales, Australia

${ }^{7}$ Department of General Practice, Monash University, Melbourne, Victoria, Australia

${ }^{8}$ Department of Obstetrics and Gynecology, School of Medicine, Indiana University, Indianapolis, Indiana, USA

Acknowledgements The authors thank Dr Maria de Leon-Santiago from the Department of General Practice, Monash University, who provided medical writing services on behalf of the ACCORd research team.

Contributors DM has led the development of this proposal. DM, KB, AT, JL, $\mathrm{KM}, \mathrm{MH}$ and JFP were involved with developing the study protocol, design (adapted from the US Contraceptive CHOICE study) and funding proposal. KM provided the support for the development of the statistical analyses plan. DM, $\mathrm{KB}, \mathrm{AT}, \mathrm{JL}, \mathrm{KM}, \mathrm{MH}, \mathrm{HM}$ and JFP have been involved with reviewing and editing the manuscript and have read and approved the final version.

Funding ACCORd is funded by the National Health and Medical Research Council-NHMRC grant ID: 1081743.

Competing interests None declared.

Ethics approval Ethics approval and consent to participate phase 1 of the study (the recruitment and training of GPs) and phase 2 of the study (the recruitment of women) have been approved by the Monash University Human Research Ethics Committee: CF14/3990-2014002066 and CF16/188-2016000080.

Provenance and peer review Not commissioned; externally peer reviewed.

Data sharing statement This article is distributed under the terms of the Creative Commons Attribution 4.0 International License (http:// creativecommons.org/licenses/by/4.0/), which permits unrestricted use, distribution and reproduction in any medium, provided appropriate credit and citation is given to the original authors and the source, use is non-commercial, a link to the Creative Commons license is provided, and if changes were made this is indicated. The Creative Commons Public Domain Dedication waiver (http://creativecommons.org/publicdomain/zero/1.0/) applies to the data made available in this article, unless otherwise stated.

Open Access This is an Open Access article distributed in accordance with the Creative Commons Attribution Non Commercial (CC BY-NC 4.0) license, 
which permits others to distribute, remix, adapt, build upon this work noncommercially, and license their derivative works on different terms, provided the original work is properly cited and the use is non-commercial. See: http:// creativecommons.org/licenses/by-nc/4.0/

\section{REFERENCES}

1. Sedgh G, Singh S, Hussain R. Intended and unintended pregnancies worldwide in 2012 and recent trends. Stud Fam Plann 2014;45:301-14.

2. Finer LB, Zolna MR. Unintended pregnancy in the United States: incidence and disparities, 2006. Contraception 2011;84:478-85.

3. Carson C, Kelly Y, Kurinczuk JJ, et al. Effect of pregnancy planning and fertility treatment on cognitive outcomes in children at ages 3 and 5: longitudinal cohort study. BMJ 2011;343:d4473.

4. Marie Stopes International Australia. Real choices: women, contraception and unplanned pregnancy. Melbourne, 2008.

5. Thomas CM, Cameron S. Can we reduce costs and prevent more unintended pregnancies? A cost of illness and cost-effectiveness study comparing two methods of EHC. BMJ Open 2013;3:e003815.

6. Monea E, Thomas A. Unintended pregnancy and taxpayer spending Perspect Sex Reprod Health 2011;43:88-93.

7. Trussell J. Understanding contraceptive failure. Best Pract Res Clin Obstet Gynecol 2009;23:199-209.

8. Finer LB, Henshaw SK. Disparities in rates of unintended pregnancy in the United States, 1994 and 2001. Perspect Sex Reprod Health 2006;38:90-6.

9. Winner B, Peipert JF, Zhao Q, et al. Effectiveness of long-acting reversible contraception. N Engl J Med 2012;366:1998-2007.

10. Mavranezouli I, LARC Guideline Development Group. The cost-effectiveness of long-acting reversible contraceptive methods in the UK: analysis based on a decision-analytic model developed for a National Institute for Health and Clinical Excellence (NICE) clinical practice guideline. Hum Reprod 2008;23:1338-45.

11. Trussell J, Lalla AM, Doan QV, et al. Cost effectiveness of contraceptives in the United States. Contraception 2009;79:5-14.

12. Trussell J, Henry N, Hassan F, et al. Burden of unintended pregnancy in the United States: potential savings with increased use of long-acting reversible contraception. Contraception 2013;87:154-61.

13. Spies EL, Askelson NM, Gelman E, et al. Young women's knowledge, attitudes, and behaviors related to long-acting reversible contraceptives. Womens Health Issues 2010;20:394-9.

14. Teal SB, Romer SE. Awareness of long-acting reversible contraception among teens and young adults. J Adolesc Health 2013;52(4 Suppl):S35-9.

15. Russo JA, Miller E, Gold MA. Myths and misconceptions about long-acting reversible contraception (LARC). J Adolesc Health 2013;52(4 Suppl):S14-21.

16. Black KI, Sakhaei T, Garland SM. A study investigating obstetricians and gynaecologists' management of women requesting an intrauterine device. Aust NZ J Obstet Gynaecol 2010;50:184-8.

17. Alnakash $\mathrm{AH}$. Influence of IUD perceptions on method discontinuation. Contraception 2008;78:290-3.

18. Wellings $\mathrm{K}$, Zhihong $\mathrm{Z}$, Krentel $\mathrm{A}$, et al. Attitudes towards long-acting reversible methods of contraception in general practice in the UK. Contraception 2007;76:208-14.

19. Mazza D, Harrison C, Taft A, et al. Current contraceptive management in Australian general practice: an analysis of $\mathrm{BEACH}$ data. Med J Aust 2012;197:110-14.

20. Middleton AJ, Naish J, Singer N. General practitioners' views on the use of the levonorgestrel-releasing intrauterine system in young, nulligravid women, in London, UK. Eur J Contracept Reprod Health 2011;16:311-18

21. National Collaborating Centre for Women's and Children's Health. Long-acting reversible contraception: the effective and appropriate use of long-acting reversible contraception. London: RCOG Press, 2005.

22. Black K, Lotke $\mathrm{P}$, Buhling KJ, et al., Intrauterine contraception for Nulliparous women: Translating Research into Action (INTRA) group. A review of barriers and myths preventing the more widespread use of intrauterine contraception in nulliparous women. Eur J Contracept Reprod Health Care 2012;17:340-50.

23. Bateson D, Harvey C, Williams J, et al. Intrauterine contraception: why are so few Australian women using this effective method? Med $J$ Aust 2011;194:324.
24. Arrowsmith ME, Aicken CR, Saxena S, et al. Strategies for improving the acceptability and acceptance of the copper intrauterine device. Cochrane Database Syst Rev 2012;(3): CD008896.

25. Bitzer J, Gemzell-Danielsson K, Roumen F, et al. The CHOICE study: effect of counselling on the selection of combined hormonal contraceptive methods in 11 countries. Eur J Contracept Reprod Health Care 2012;17:65.

26. Grentzer J, McNicholas C, Peipert JF. Use of the etonogestrelreleasing contraceptive implant. Expert Rev Obstet Gynecol 2013;8:337.

27. Rose SB, Lawton BA, Brown SA. Uptake and adherence to long-acting reversible contraception post-abortion. Contraception 2010;82:345-53.

28. Rose SB, Lawton BA. Impact of long-acting reversible contraception on return for repeat abortion. Am J Obstet Gynecol 2012;206:37. e31-6.

29. Secura GM, Allsworth JE, Madden T, et al. The Contraceptive CHOICE Project: reducing barriers to long-acting reversible contraception. Am J Obstet Gynecol 2010;203:115.e111-17.

30. Peipert JF, Madden T, Allsworth JE, et al. Preventing unintended pregnancies by providing no-cost contraception. Obstet Gynecol 2012;120:1291-7.

31. O'Neil-Callahan M, Peipert JF, Zhao Q, et al. Twenty-four-month continuation of reversible contraception. Obstet Gynecol 2013;122:1083-91.

32. Harper CC, Rocca $\mathrm{CH}$, Thompson KM, et al. Reductions in pregnancy rates in the USA with long-acting reversible contraception: a cluster randomised trial. Lancet 2015;386: 562-8.

33. Sexual Health and Family Planning Australia. Time for a change: increasing the use of long acting reversible contraceptive methods in Australia. Canberra, 2013.

34. Richters J, Grulich AE, de Visser RO, et al. Sex in Australia: contraceptive practices among a representative sample of women. Aust N Z J Public Health 2003;27:210-16.

35. Gray $E$, McDonald $P$. Using a reproductive life course approach to understand contraceptive method use in Australia. J Biosoc Sci 2010;42:43-57.

36. Campbell MK, Elbourne DR, Altman DG, CONSORT Group. CONSORT statement: extension to cluster randomised trials. BMJ 2004;328:702-8.

37. Piantadosi S. Clinical trials: a methodologic perspective. 2nd edn. John Wiley \& Sons Inc, NJ, USA: 2005.

38. Broughton HO, Omvig KJ, Buckel CM, et al. LARC First. 2013 (cited 13 Jul 2015). http://www.larcfirst.com/index.html

39. Madden T, Mullersman JL, Omvig KJ, et al. Structured contraceptive counseling provided by the Contraceptive CHOICE Project. Contraception 2013;88:243-9.

40. The Royal Australian College of General Practitioners (RACGP). Guidelines for Preventive Activities in General Practice. 2012 (cited in 13 Jul 2015); 8th. http://www.racgp.org.au/your-practice/ guidelines/redbook/

41. Faculty of Sexual and Reproductive Healthcare Clinical Effectiveness Unit. Faculty of sexual and reproductive healthcare clinical guidance: quick starting contraception. London: Faculty of Sexual and Reproductive Healthcare, 2010.

42. Osborne RH, Batterham RW, Elsworth GR, et al. The grounded psychometric development and initial validation of the Health Literacy Questionnaire (HLQ). BMC Public Health 2013;13:658.

43. Pawson R, Tilley N. Realistic evaluation. London: SAGE Publications Ltd., 1997.

44. Hegarty K, O'Doherty L, Taft A, et al. Screening and counselling in the primary care setting for women who have experienced intimate partner violence (WEAVE): a cluster randomised controlled trial. Lancet 2013;382:249-58.

45. Knox SA, Chondros P. Observed intra-cluster correlation coefficients in a cluster survey sample of patient encounters in general practice in Australia. BMC Med Res Methodol 2004;4:30.

46. Donner A, Klar N. Design and analysis of cluster randomisation trials in health research. London: Hodder Arnold, 2000.

47. Brazier J, Roberts J, Deverill M. The estimation of a preference-based measure of health from the SF-36. J Health Econ 2002;21:271-92. 